\title{
Dancing Fascicles - A New Signal Related to Hepatitis B Immunization
}

\author{
B. Adhisivam • B. Vishnu Bhat
}

Received: 7 July 2014 / Accepted: 4 November 2014 / Published online: 23 November 2014

(C) Dr. K C Chaudhuri Foundation 2014

To the Editor: Any untoward medical occurrence which follows immunization and which does not necessarily have a causal relationship with the usage of the vaccine is defined as anadverse event following immunization (AEFI) [1]. We report here a curious AEFI with Hepatitis $B$ vaccine in a newborn. Aterm male baby weighing $2.06 \mathrm{~kg}$ born by spontaneous vaginal delivery to a primi mother after a normal antenatal period was noted to have rippling movements beneath the skin over left thigh following routine intramuscular injection of Hepatitis B vaccine at the anterolateral thigh $1 \mathrm{~h}$ after birth. On close observation, muscle fasciculations were noted in the same thigh which persisted for $3 \mathrm{~h}$ and then subsided spontaneously. The newborn was under the radiant warmer and euthermic. He was hemodynamically stable and feeding well. These fasciculations were localized and there was no clinical evidence of jitteriness or seizures. The tone of the baby was normal and there were no tongue fasciculations. His blood sugar and serum levels of calcium, magnesium, sodium and creatinine phospho kinase (CPK) were normal. There was a temporal relationship between the fasciculations and the intramuscular injection of Hepatitis B vaccine. The mechanical trauma of injection or the vaccine per se could have caused the fasciculations. All the other newborns who received the same batch of vaccine on that day did not show similar symptoms. As this new-

B. Adhisivam $(\bowtie) \cdot$ B. V. Bhat

Division of Neonatology, Department of Pediatrics, Jawaharlal

Institute of Postgraduate Medical Education and Research

(JIPMER), Pondicherry 605 006, India

e-mail: adhisivam1975@yahoo.co.uk born was neurologically normal, the fasciculations were probably benign but certainly qualify for AEFI and could be a new "signal" as per pharmacovigilance terminology. A personal communication with Uppsala Monitoring Centre, Sweden revealed that there is only one such report in a newborn as per VigiBase.

The infant was found neurologically normal when he was brought for immunization at 6,10 and $14 \mathrm{wk}$.

Fasciculations or "muscle twitches", are small, local, involuntary muscle contractions and relaxations which may be visible under the skin. They arise as a result of spontaneous depolarization of a lower motor neuron leading to the synchronous contraction of all the skeletal muscle fibers within a single motor unit. Majority of the fasciculations are benign, but can also be due to disease of the motor neurons. Fatigue, anxiety, stress and metabolic causes including calcium, magnesium and sodium chloride may cause fasciculations. Fasciculations in newborn following Hepatitis B vaccine are rare and this new signal should be closely monitored.

Conflict of Interest None.

Source of Funding None.

\section{Reference}

1. World Health Organization. Immunization Safety Surveillance Guidelines for Immunization Programme Managers on Surveillance of Adverse Events Following Immunization. 2nd ed. Western Pacific: WHO Press; 2013:9. Available at: http:// www.wpr.who.int/topics/immunization_safety/ ImmunizationSafetySurveillance.pdf. Accessed 27 Sep 2013. 